ISAHP 2007, Viña Del Mar, Chile, August 3-8, 2007

\title{
USING ANALYTIC NETWORK PROCESS MODEL IN COMBINATORIAL AUCTIONS ${ }^{1}$
}

\author{
Petr Fiala \\ Department of Econometrics, University of Economics \\ Praha, 130 67, Czech Republic \\ pfiala@vse.cz
}

Keywords: Combinatorial auctions, preference elicitation, Analytic Network Process, Dynamic Network Process

Summary: Auctions are important market mechanisms for the allocation of goods and services. Auctions are preferred often to other common processes because they are open, quite fair, easiness to understand by participants, and lead to economically efficient outcomes. Design of auctions is a multidisciplinary effort made of contributions from economics, operations research, informatics, and other disciplines. Combinatorial auctions are those auctions in which bidders can place bids on combinations of items, called "packages," rather than just individual items. The advantage of combinatorial auctions is that the bidder can more fully express his preferences. This is particular important when items are complements. The multiple evaluation criteria can be used. There are dependencies among sellers, buyers, criteria, bundles of items. A variety of feedback processes creates complex system of items. For the whole structure seems to be very appropriate Analytic Network Process (ANP) approach. The ANP method makes possible to deal systematically with all kinds of dependence and feedback in the system of items. By the ANP approach can be evaluated the preferences of bundles of items. Dynamic Network Process (DNP) as an extension of ANP can deal with time dependent priorities in combinatorial auctions.

\section{Introduction}

Auctions are important market mechanisms for the allocation of goods and services. Many modern markets are organized as auctions. Combinatorial auctions are those auctions in which bidders can place bids on combinations of items, so called bundles. The advantage of combinatorial auctions is that the bidder can more fully express his preferences. This is particular important when items are complements. The auction designer also derives value from combinatorial auctions. Allowing bidders more fully to express preferences often leads to improved economic efficiency and greater auction overall valuation. However, alongside their advantages, combinatorial auctions raise a host of questions and challenges (see Cramton et al. 2006, Rothkopf et al. 1998).

Auction theory has caught tremendous interest from both the economic side as well as the Internet industry. An auction is a competitive mechanism to allocate resources to buyers based on predefined rules. These rules define the bidding process, how the winner is determined, and the final agreement. In electronic commerce transactions, software agents that negotiate on behalf of buyers and sellers conduct auctions. The popularity of auctions and the requirements of e-business have led to growing interest in the development of complex trading models (see Bellosta et al. 2004, Bichler 2000, Oliveira et al. 1999).

Classification of auctions is based on some specific characteristics as:

\footnotetext{
${ }^{1}$ The paper is supported by the Grant Agency of Czech Republic (grant 402/07/0166 „Combinatorial auctions - modeling and analysis“).
} 
- the numbers of sellers and buyers,

- the number of items,

- traded items (indivisible, divisible, pure commodities, structured commodities),

- participants' roles in auctions (one-sided, multilateral auctions),

- preferences of the participants,

- the form of the private information participants have about preference,

- objectives of auctions (optimization, allocation rules, pricing rules),

- evaluating criteria,

- complexity of bids (simply, related bids),

- organization of auctions (single-round, multi-round, sequential, parallel, price schemes).

Many specific factors of combinatorial auctions can be modeled and analyzed by Analytic Network Process and Dynamic Network Process (ANP/DNP) methods.

\section{Multicriteria combinatorial auctions}

For our model, we propose to use multidimensional auctions. These auctions can be classified:

- multi-unit auction,

- multi-item auction,

- multi-criteria auction,

Multi-unit auctions contain multiple units of items and makes possible volume discount auction. Multiitem auctions can place bids on combinations of items, so called combinatorial auctions. In the combinatorial auctions can be defined multiple criteria as

- revenue maximization - the seller should extract the highest possible price,

- efficiency - the buyers with the highest valuation get the goods,

- collusion possibility,

Auctions with complex bid structures are also called multicriteria auctions, since they address multiple attributes of the items (quality, quantity, price) in the negotiation space. Multicriteria optimization can be helpful for detailed analysis of combinatorial auctions. Buyers can specify aspiration levels that express their desired values on the attributes of the items to be purchased and reservation levels that represent the minimal values required. There are possible combinations of the multidimensional characteristics as multi-criteria, multi-unit, multi-item auctions.

Multicriteria auctions require several key components to automate the process:

- a preference model,

- a multicriteria optimization model,

- a negotiation model.

The preference model is used to let the buyer express his preferences. The multicriteria optimization model selects the best offer for the buyer. The negotiation model helps to find a consensus by auctions (Fiala, 1997).

\section{Winner determination problem}

The problem, called the winner determination problem, has received considerable attention in the literature. The problem is formulated as: Given a set of bids in a combinatorial auction, find an allocation of items to bidders that maximizes the seller's revenue. It was introduced many important ideas, such as the mathematical programming formulation of the winner determination problem, the connection between the winner determination problem and the set packing problem as well as the issue of complexity. There 
are some different approaches to solve winner determination problems. Main approaches for solving the problem are exact or approximate algorithms. The algorithms have some pros and cons. We propose to use an iterative approach for solving winner determination problems. The iterative approach is based on a primal-dual algorithm.

\section{Problem formulation}

Many types of combinatorial auctions can be formulated as mathematical programming problems. From different types of combinatorial auctions we present an auction of indivisible items with one seller and several buyers. Let us suppose that one seller offers a set $G$ of $m$ items, $j=1,2, \ldots, m$, to $n$ potential buyers. Items are available in single units. A bid made by buyer $i, i=1,2, \ldots, n$, is defined as

where

$$
B_{i}=\left\{S, v_{i}(S)\right\},
$$

$S \subseteq M$, is a combination of items,

$v_{i}(S)$, is the valuation by buyer $i$ for the combination of items $S$, the valuation is made by multiple criteria.

The objective is to maximize the overall valuation of the seller given the bids made by buyers. Constraints establish that no single item is allocated to more than one buyer and that no buyer obtains more than one combination.

Bivalent variables are introduced for model formulation:

$x_{i}(S)$ is a bivalent variable specifying if the combination $S$ is assigned to buyer $i\left(x_{i}(S)=1\right)$.

The winner determination problem can be formulated as follows

$$
\sum_{i=1}^{n} \sum_{S \subseteq M} v_{i}(S) x_{i}(S) \rightarrow \quad \max
$$

subject to

$$
\begin{aligned}
& \sum_{S \subseteq M} x_{i}(S) \leqq 1, \forall i, i=1,2, \ldots, n, \\
& \sum_{i=1}^{n} \sum_{S \subseteq M} x_{i}(S) \leqq 1, \forall j \in M, \\
& x_{i}(S) \in\{0,1\}, \forall S \subseteq M, \forall i, i=1,2, \ldots, n .
\end{aligned}
$$

The objective function expresses the overall valuation. The first constraint ensures that no bidder receives more than one combination of items. The second constraint ensures that overlapping sets of items are never assigned.

The winner determination problem, i.e., determining the items that each bidder wins is not difficult in the case of non-combinatorial auctions. It would take $O(n m)$ time where $n$ is the number of bidders and $m$ is the number of items. But in the case of combinatorial auctions, the winner determination problem is much more complex. The payment determination problem also is an important and non-trivial problem.

\section{Complexity of the problem}

Complexity is a fundamental question in combinatorial auction design. There are some types of complexity: 
- computational complexity,

- valuation complexity,

- strategic complexity,

- communication complexity.

Computational Complexity covers such questions: How much computation is expected of the mechanism to compute an outcome given the bid information of the bidders. This is an extremely important question because winner determination problem is an NP-complete optimization problem. The winner determination problem turns out to be an instance of a weighted set packing problem. The weighted set packing problem is a problem of finding a disjoint collection of weighted subsets of a larger set with maximal total weight. Weighted set packing is a classical NP-complete problem.

Valuation complexity deals with such questions: How much computation is required to provide preference information within a mechanism? Estimating every possible bundle of items requires exponential space and hence exponential time. Bidders need to determine valuations for $2^{m}-1$ possible bundles.

Strategic complexity concerns such questions: Which of the $2^{m}-1$ bundles to bid on? What is the best strategy for bidding? Must bidders model behavior of other bidders and solve problems to compute an optimal strategy? For instance, in a sealed bid combinatorial procurement scenario, sellers will need to not only take their valuation of the bundles into consideration but also the bidding behavior of their competitors. This requires sophisticated bidding logic.

Communication complexity concerns such questions: How much communication is required to exchange between bidders and auctioneer until an equilibrium price is reached the mechanism to compute an outcome. The amount of communication between the bidders and the auctioneer can become quite high. For instance, in an iterative combinatorial auction, where individual valuations are revealed progressively in an iterative manner, the communication costs could be high if the auction were conducted in a distributed manner over space and/or time. The problem of communication complexity can be addressed through the deign of careful bidding languages that provide expressive but concise bids.

\section{Solving the problem}

The algorithms proposed for solving the winner determination problem fall into two classes:

- exact algorithms, and

- approximate algorithms.

Exact approaches to solving the winner determination problem require algorithms that generate both good lower and upper bounds on the maximum objective function value. In general, the upper bound on the optimal solution value is obtained by solving a relaxation of the optimization problem. There are two standard relaxations for winner determination problem: Lagrangean relaxation and the linear programming relaxation. By Lagrangean relaxation the feasible set is usually required to maintain $0-1$ feasibility, but many if not all of the constraints are moved to the objective function with a penalty term. By LP relaxation only the integrality constraints are relaxed, the objective function remains the original function. Exact methods come in three varieties: branch and bound, cutting planes and a hybrid called branch and cut. Integer programming techniques can be used to handle winner determination in combinatorial auctions with a small enough number of items.

There are a few special cases where the winner determination problem can be solved exactly using polynomial time algorithms. For example, paper (de Vries and Vohra. 2003) looks at polynomially solvable instances of the winner determination problem in terms of the structure of the constraint matrix: totally unimodular matrices, balanced matrices, and perfect matrices. A matrix is said to be totally unimodular if the determinant of every square submatrix is 0,1 , or -1 . A $0-1$ matrix is called balanced if it has no submatrix of odd order with exactly two 1's in each row and column. A matrix is said to be 
perfect if it is the vertex-clique adjacency matrix of a perfect graph. In all these cases, it is shown that the winner determination problem can be solved as a linear program.

Approximate algorithms have also emerged as a major approach to solving the allocation problem in combinatorial auctions. One way of dealing with hard integer programs is to give up on finding the optimal solution. An approximation algorithm is a polynomial time algorithm with a provable performance guarantee. Approximate algorithm seeks a feasible solution fast and hopes that it is near optimal. Fast approximation algorithms for the winner determination problem can be obtained by translating results for combinatorial problems related to winner determination, mainly the weighted set packing problem and the weighted stable set problem to the context of winner determination (see Sandholm 2002). Approximate algorithms and heuristics include greedy algorithms, simulated annealing, genetic algorithms and others.

\section{Preference elicitation}

The key feature that makes combinatorial auctions most appealing is the ability for bidders to express complex preferences over bundles of items, involving complementarity and substitutability. Items are complements when a set of items has greater utility than the sum of the utilities for the individual items. Items are substitutes when a set of items has less utility than the sum of the utilities for the individual items.

Two items $A$ and $B$ are complementary, if it holds

$$
v(\{A, B\})>v(\{A\})+v(\{B\}) .
$$

Two items $A$ and $B$ are substitute, if it holds

$$
v(\{A, B\})<v(\{A\})+v(\{B\}) .
$$

Different elicitation algorithms may require different means of representing the information obtained by bidders, Sandholm and Boutilier (2006) describe a general method for representing an incompletely specified valuation functions. A constraint network is a labeled directed graph consisting of one node for each bundle $b$ representing the elicitor's knowledge of the preferences of a bidder. A directed edge $(a, b)$ indicates that bundle a is preferred to bundle $b$. Figure 1 represents an example of a constraint network for bundles of three items $(\mathrm{A}, \mathrm{B}, \mathrm{C})$.

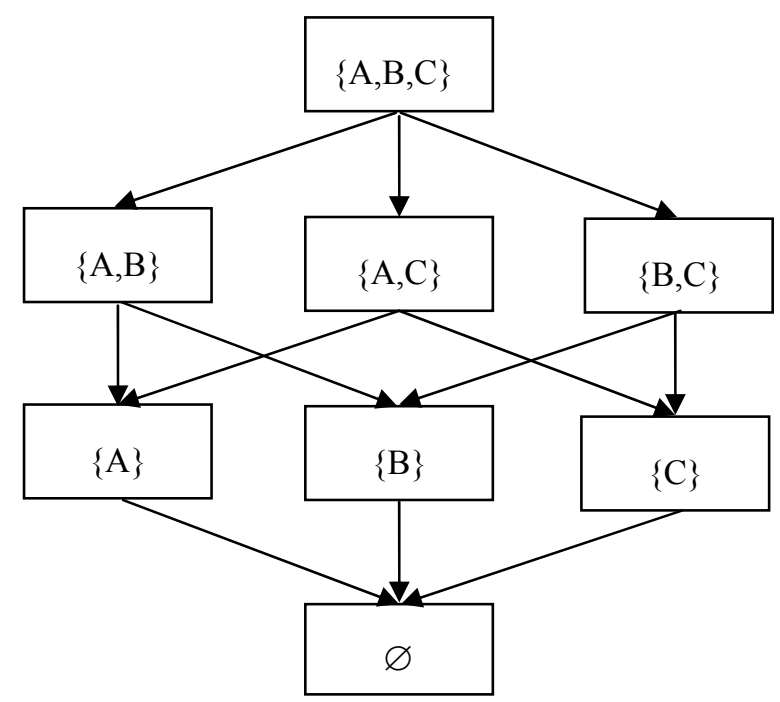

Figure 1 Constraint network 
The constraint network representation is useful conceptually, and can be represented explicitly for use in various elicitation algorithms. But its explicit representation is generally tractable only for small problems, since it contains $2^{m}$ nodes. For preference elicitation of bundles in a constraint network can be used Analytic Network Process.

The Analytic Hierarchy Process (AHP) is the method for setting priorities (Saaty, 1996). A priority scale based on reference is the AHP way to standardize non-unique scales in order to combine multiple performance measures. The AHP derives ratio scale priorities by making paired comparisons of elements on a common hierarchy level by using a 1 to 9 scale of absolute numbers. The absolute number from the scale is an approximation to the ratio $\mathrm{w}_{\mathrm{j}} / \mathrm{w}_{\mathrm{k}}$ and then is possible to derive values of $\mathrm{w}_{\mathrm{j}}$ and $\mathrm{w}_{\mathrm{k}}$. The AHP method uses the general model for synthesis of the performance measures in the hierarchical structure.

$$
u_{i}=\sum_{j=1}^{n} v_{j} w_{j k} .
$$

The Analytic Network Process (ANP) is the method (Saaty, 2001) that makes it possible to deal systematically with all kinds of dependence and feedback in the performance system. The well-known AHP theory is a special case of the Analytic Network Process that can be very useful for incorporating linkages in the system.

The structure of the ANP model is described by clusters of elements connected by their dependence on one another. A cluster groups elements that share a set of attributes. At least one element in each of these clusters is connected to some element in another cluster. These connections indicate the flow of influence between the elements ( see Figure 2).

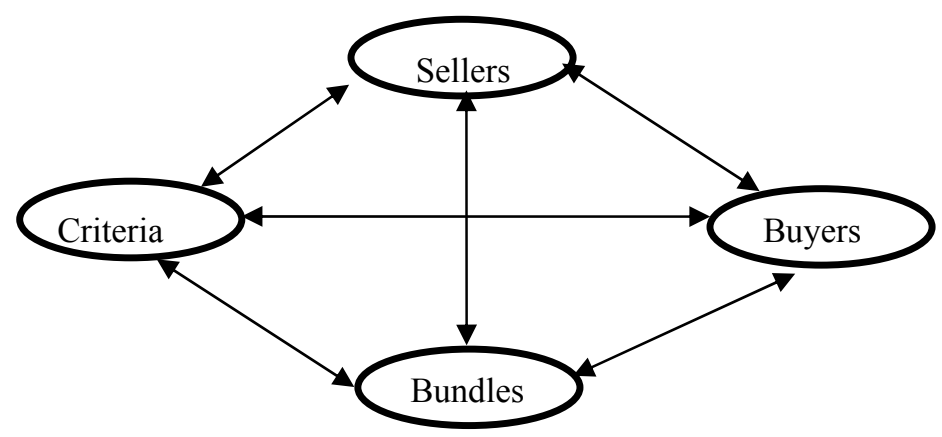

Figure 2 Clusters and connections in multicriteria combinatorial auctions

The clusters in multicriteria combinatorial auctions can be sellers, buyers, bundles of items, and evaluating criteria also. Paired comparisons are inputs for preference elicitation in combinatorial auctions. A supermatrix is a matrix of all elements by all elements. The weights from the paired comparisons are placed in the appropriate column of the supermatrix. The sum of each column corresponds to the number of comparison sets. The weights in the column corresponding to the cluster are multiplied by the weight of the cluster. Each column of the weighted supermatrix sums to one and the matrix is column stochastic. Its powers can stabilize after some iterations to limited supermatrix. The columns of each block of the matrix are identical in many cases, though not always, and we can read off the global priority of units.

Recent work has focused on the question of how to limit the amount of valuation information provided by bidders by adaptively limiting the precision of the bids that are specified. 


\section{Iterative combinatorial auctions}

In the iterative approach, there are multiple rounds of bidding and allocation and the problem is solved in an iterative and incremental way. Iterative combinatorial auctions with multiple criteria are proposed in the paper as complex trading models.

One way of reducing some of the computational burden in solving the winner determination problem is to set up a fictitious market that will determine an allocation and prices in a decentralized way. In the iterative approach, there are multiple rounds of bidding and allocation and the problem is solved in an iterative and incremental way. Iterative combinatorial auctions are attractive to bidders because they learn about their rivals' valuations through the bidding process, which could help them to adjust their own bids. For analysis of iterative combinatorial auctions we propose to use dynamic version of ANP.

Auctions have emerged as a particularly interesting tool for negotiations. Combinatorial auctions provide a mechanism for negotiation between buyers and sellers. Various concepts of negotiation models can be used for modeling of combinatorial auctions.

There is a connection between efficient auctions for many items, and duality theory. The Vickrey auction can be taken as an efficient pricing equilibrium, which corresponds to the optimal solution of a particular linear programming problem and its dual. The simplex algorithm can be taken as static approach to determining the Vickrey outcome. Alternatively, the primal-dual algorithm can be taken as a decentralized and dynamic method of determine the pricing equilibrium.

A primal-dual algorithm usually maintains a feasible dual solution and tries to compute a primal solution that is both feasible and satisfies the complementary slackness conditions. If such a solution is found, the algorithm terminates. Otherwise the dual solution is updated towards optimality and the algorithm continues with the next iteration.

The fundamental work (Bikhchandani and Ostroy 2002) demonstrates a strong interrelationship between the iterative auctions and the primal-dual linear programming algorithms. A primal-dual linear programming algorithm can be interpreted as an auction where the dual variables represent item prices. The algorithm maintains a feasible allocation and a price set and terminates as the efficient allocation and competitive equilibrium prices are found.

For the winner determination problem we will formulate the LP relaxation and its dual. Consider the LP relaxation of the winner determination problem (1):

$$
\sum_{i=1}^{n} \sum_{S \subseteq M} v_{i}(S) x_{i}(S) \rightarrow \quad \max
$$

subject to

$$
\begin{aligned}
& \sum_{S \subseteq M} x_{i}(S) \leqq 1, \forall i, i=1,2, \ldots, n, \\
& \sum_{i=1}^{n} \sum_{S \subseteq M} x_{i}(S) \leqq 1, \forall j \in M, \\
& x_{i}(S) \geq 0, \forall S \subseteq M, \forall i, i=1,2, \ldots, n .
\end{aligned}
$$

The corresponding dual to problem (2) 


$$
\sum_{i=1}^{n} p(i)+\sum_{j \in S} p(j) \rightarrow \quad \min
$$

subject to

$$
\begin{aligned}
p(i)+\sum_{j \in S} p(j) \geq v_{i}(S) & \forall i, S, \\
p(i), p(j) \geq 0, & \forall i, j,
\end{aligned}
$$

The dual variables $p(j)$ can be interpreted as anonymous linear prices of items, the term $\sum_{j \in S} p(j)$ is then the price of the bundle $S$ and $p(i)=\max _{S}\left[v_{i}(S)-\sum_{j \in S} p(j)\right]$ is the maximal utility for the bidder $i$ at the prices $p(j)$.

Several auction formats based on the primal-dual approach have been proposed in the literature. Though these auctions differ in several aspects, the general scheme can be outlined as follows:

1. Choose minimal initial prices.

2. Announce current prices and collect bids. Bids have to be higher or equal than the prices.

3. Compute the current dual solution by interpreting the prices as dual variables. Try to find a feasible allocation, an integer primal solution that satisfies the stopping rule. If such solution is found, stop and use it as the final allocation. Otherwise update prices and go back to 2 .

Concrete auction formats based on this scheme can be implemented in different ways. The most important design choices are the following:

- pricing scheme,

- $\quad$ price update rule,

- way of computing a feasible primal solution in each iteration,

- stopping rule,

- type of information feedback.

The combinatorial auctions can be classified into the auctioneer-side allocation auctions and the bidderside allocation auctions. The bidder-side allocation auctions were developed for small problems where bidders can cooperate in order to find a better allocation by themselves in each iteration. In the auctioneer-side allocation auctions the auctioneer solves the winner determination problem after the bids are collected. The auctioneer then provides some kind of feedback to support the bidders in improving their bids in the next iteration. Usually the bidder's current winning bids and item prices are used as the feedback. The key challenge in the iterative combinatorial auction design is to provide information feedback to the bidders after each iteration. Assigning prices to items and/or item bundles was adopted as the most intuitive mechanism of providing feedback.

The AHP and ANP have been static but for today's world analyzing is very important time dependent decision making. The DHP/DNP (Dynamic Hierarchy Process/ Dynamic Network Process) methods were introduced (Saaty, 2003). There are two ways to study dynamic decisions: structural, by including scenarios, and functional by explicitly involving time in the judgment process. For the functional dynamics there are analytic or numerical solutions. The basic idea with the numerical approach is to obtain the time dependent principal eigenvector by simulation.

The Dynamic Network Process seems to be the appropriate instrument for analyzing dynamic network effects (Fiala, 2006). The method is appropriate also for the specific features of multicriteria combinatorial auctions. The method computes time dependent weights for bundles of items of weights of bidders (Figure 3 ). 


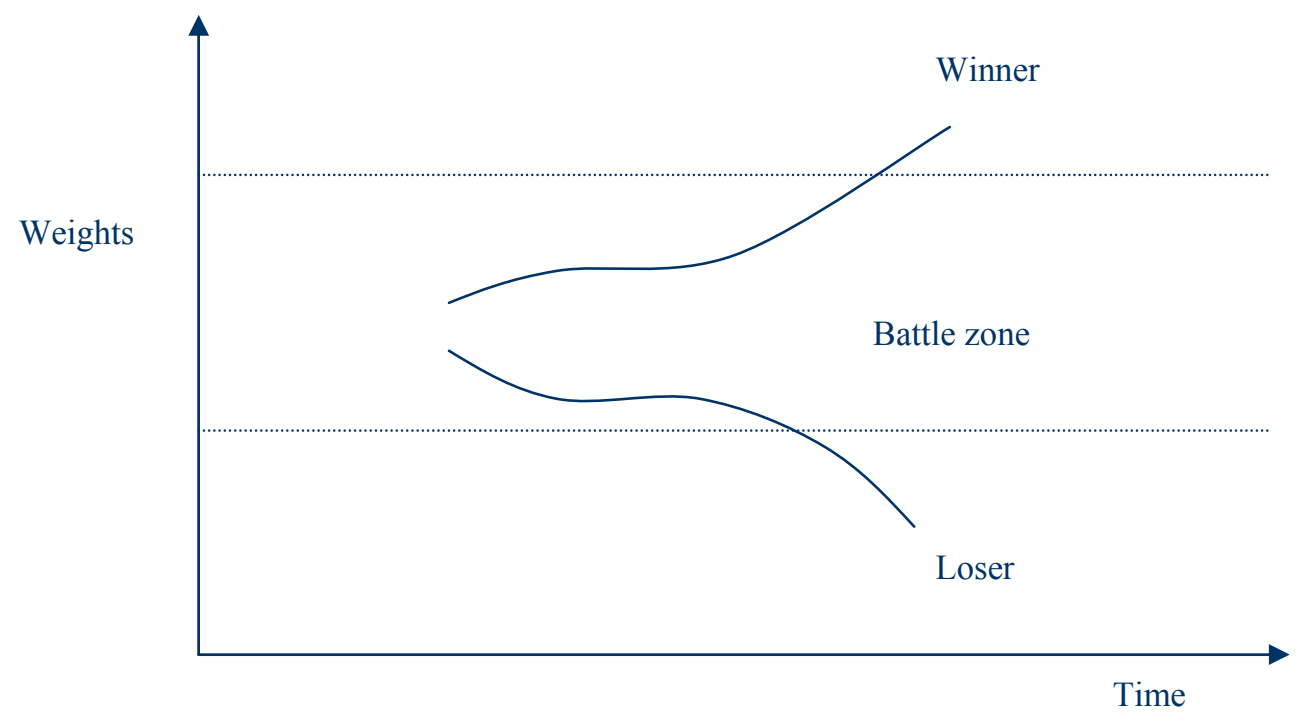

Figure 3 Time dependent weights

In the multicriteria combinatorial auction model we take into account auctioneer, bidders, criteria and packages as clusters and different types of connections in the system. There are some dependencies and feedback among elements and clusters also. The dynamic version of the model is tested.

We used the alpha version of the ANP software Super Decisions developed by Creative Decisions Foundation (CDF) for some experiments for testing the possibilities of the expression and evaluation of the multicriteria combinatorial auction models (Figure 4). 


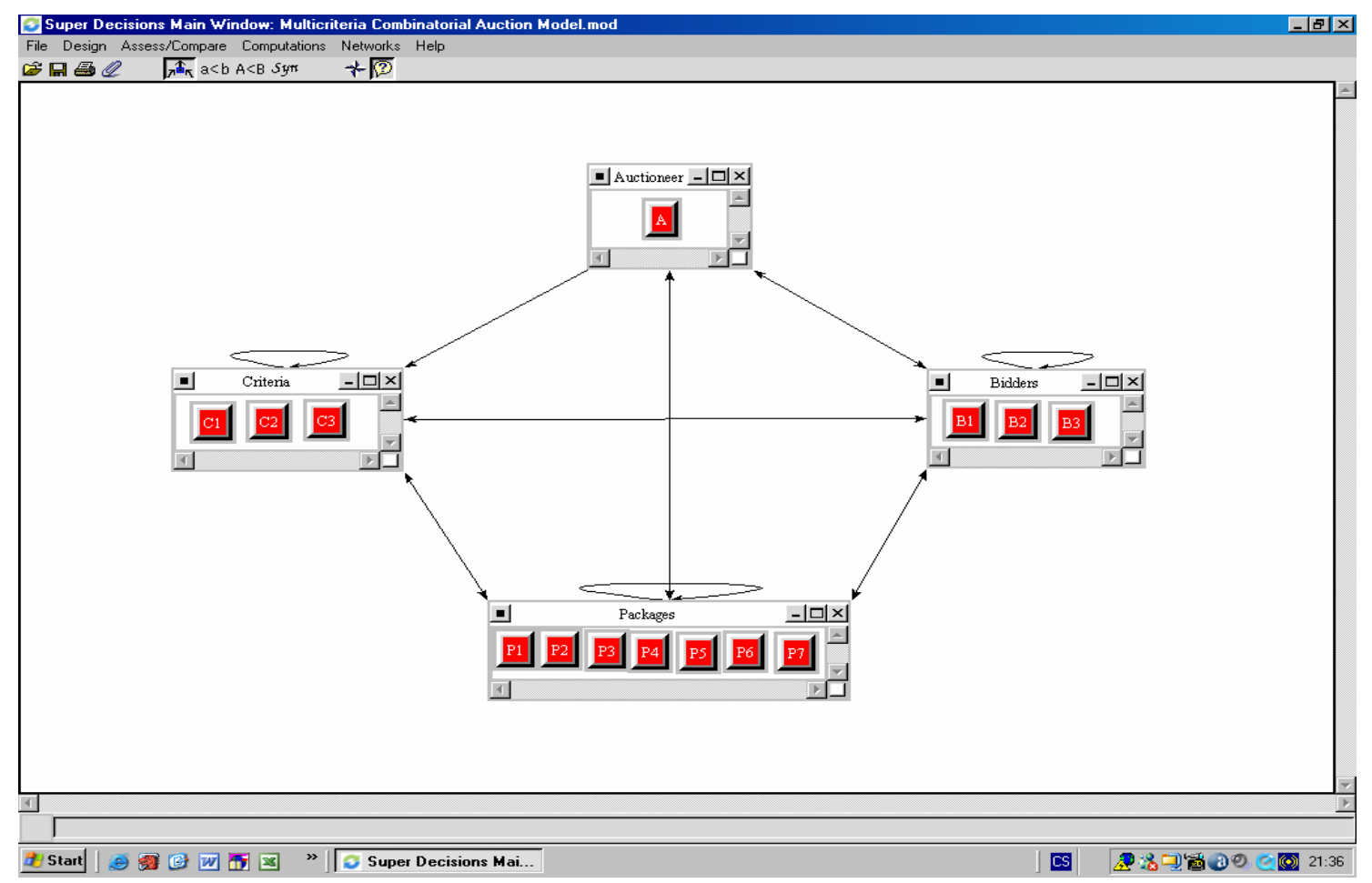

\section{Conclusions}

Figure 4 Multicriteria Combinatorial Auction Model

For electronic auctions we propose to use multicriteria iterative combinatorial auctions. Combinatorial auction is the important subject of an intensive economic research. Iterative process helps the bidders express their preferences. Combinatorial auctions promise to increase efficiency and reduce exposure risk in an economic environment where synergy is significant. The winner determination problem is by far the most researched issue in combinatorial auctions. Winner determination problem is an NP-complete optimization problem. Decentralized way for iterative combinatorial auctions based on primal-dual algorithm seems to be very promising for solving the winner determination problem. There are many variations how to use this approach. Multicriteria optimization can be helpful for detailed analysis of combinatorial auctions. The combination of such approaches can give more complex views on auctions. A possible flexible approach is presented. The approach is based on the Dynamic Network Process.

\section{References}

Bellosta M, Brigui I, Kornman S, and Vanderpooten D (2004) “A multi-criteria model for electronic auctions", ACM Symposium on Applied Computing, 759-765.

Bichler M (2000) “An experimental analysis of multi-attribute auctions,” Decision Support Systems, 29, 249- 268.

Bikhchandani S, and Ostroy JM (2002) “The package assignment model”, Journal of Economic Theory, 107, 377-406.

CDF (Creative Decisions Foundation) www page (2000)- www.creativedecisions.net.

Cramton P, Shoham Y, and Steinberg R (eds.) (2006) Combinatorial Auctions, Cambridge, MIT Press.

de Vries S, and Vohra RV (2003) “Combinatorial auctions: A survey”, INFORMS Journal of Computing, 15, 284-309, 
Fiala P (2006) "An ANP/DNP analysis of economic elements in today's world network economy", Journal of Systems Science and Systems Engineering, 15, 131-140.

Fiala P (1997) "Models of cooperative decision making", Gal T, and Fandel G (eds.) Multiple Criteria Decision Making, Springer.

Oliveira E, Fonsesca JM, and Steiger-Garao A (1999) "Multi-criteria negotiation in multi-agent systems", 1st International Workshop of Central and Eastern Europe on Multi-agent Systems (CEEMAS'99), St. Petersbourg.

Rothkopf M, Pekeč A, and Harstad R (1998) "Computationally manageable combinational auctions", Management Science, 8, 1131-1147.

Saaty TL (1996) The Analytic Hierarchy Process, Pittsburgh, RWS Publications.

Saaty TL (2001) Decision making with Dependence and Feedback: The Analytic Network Process, Pittsburgh, RWS Publications.

Saaty TL (1999) "Time Dependent Decision-Making; Dynamic Priorities in AHP/ANP: Generalizing from Points to Functions and from Real to Complex Variables", Proceedings of the $7^{\text {th }}$ International Conference on the Analytic Hierarchy Process, Bali, Indonesia, 1-38.

Sandholm T (2002) "Algorithm for optimal winner determination in combinatorial auctions", Artificial Intelligence, 135, 1-54.

Sandholm T, Boutilier C (2006) "Preference elicitation in combinatorial auctions", Cramton P, Shoham Y, and Steinberg R (eds.) Combinatorial Auctions, Cambridge, MIT Press. 